\title{
Current-injection in a ballistic multiterminal superconductor/two-dimensional electron gas Josephson junction
}

\author{
Th. Schäpers, * V. A. Guzenko, and R. P. Müller ${ }^{\dagger}$ \\ Institut für Schichten und Grenzflächen (ISG-1), Forschungszentrum Jülich, D-52425 Jülich, Germany \\ A. A. Golubov and A. Brinkman \\ Faculty of Applied Physics, University of Twente, P. O. Box 217, 7500 AE Enschede, The Netherlands \\ G. Crecelius, A. Kaluza, ${ }^{\ddagger}$ and H. Lüth \\ Institut für Schichten und Grenzflächen, Forschungszentrum Jülich, D-52425 Jülich, Germany
}

(Received 19 July 2002; published 22 January 2003)

\begin{abstract}
We study the suppression of the critical current in a multi-terminal superconductor/two-dimensional electron gas/superconductor Josephson junction by means of hot carrier injection. As a superconductor $\mathrm{Nb}$ is used, while the two-dimensional electron gas is located in a strained InGaAs/InP heterostructure. Two different modes of injection are employed. First, in the three-terminal injection mode, where the injection current flows from an injector contact to one of the superconducting electrodes, only a partial suppression is obtained. Second, in the four-terminal mode, where the injection current flows between two opposite injector contacts, a complete suppression is achieved. A theoretical model for the critical current suppression in a short junction is presented, which takes the two-dimensional character of the junction into account. Qualitatively, the experimental data agree well with the theoretical predictions. The injection voltage required in the experiment to suppress the supercurrent is lower than theoretically predicted. This is explained by the fact that the width of the normal region of the junction is slightly too large to be in the short-junction limit.
\end{abstract}

DOI: 10.1103/PhysRevB.67.014522

PACS number(s): 74.50.+r, 73.23.-b

\section{INTRODUCTION}

The Josephson supercurrent in a superconductor/normal conductor/superconductor $(S N S)$ junction can be controlled by injecting carriers into the normal conducting region. This effect was first demonstrated by Morpurgo et al. ${ }^{1}$ for a diffusive junction consisting of $\mathrm{Nb}$ as a superconductor and $\mathrm{Au}$ as a normal metal. In addition, control of a supercurrent was also observed in ballistic junctions with a high mobility twodimensional electron gas (2DEG) as an $N$ layer. $^{2-4}$ Control of a supercurrent by coupling a normal reservoir via a constriction to a ballistic $S N S$ junction was first proposed by van Wees et al. ${ }^{5}$ Further theoretical studies on ballistic nonequilibrium junctions dealt with the anomalous dc Josephson current, ${ }^{6,7}$ four-terminal junctions, ${ }^{8,9,7}$ and Andreev level spectroscopy. ${ }^{10}$ The current injection into a diffusive junction was first discussed by Volkov. ${ }^{11}$ For this type of junction even a reversal of the critical current was observed experimentally and explained theoretically. ${ }^{12,13}$ By employing a superconductor instead of a normal conductor as an injector a supercurrent enhancement was obtained for an injection voltage which corresponds to the superconducting gap. ${ }^{14}$

The Josephson effect in ballistic superconductor/normal conductor/superconductor junctions can be described within the framework of phase-coherent Andreev reflection. ${ }^{15-18}$ In the Andreev reflection process an electron incident from the normal conductor side on the normal conductor/ superconductor interface is retroreflected as a hole by creating a Cooper pair in the superconductor. ${ }^{19}$ In the reverse process, a hole is retroreflected as an electron by annihilating a Cooper pair in the superconductor. In case of an $S N S$ junction, where two superconductors are separated by a thin nor- mal conductor layer, coherent multiple Andreev reflections can occur between both interfaces. Since a Cooper pair is annihilated on one interface and created at the opposite one, or vice versa, a supercurrent is carried by this multiple Andreev reflection process. The net supercurrent in a SNS junction is constituted from the current contribution of discrete Andreev levels, energetically located within the superconducting gap $\Delta_{0}$ as well as by extended states beyond $\Delta_{0}$. The current direction of the Andreev levels depends on the phase difference between the superconducting electrodes and on the energy, i.e., the supercurrent of neighboring discrete Andreev levels flows in opposite directions. The maximum supercurrent, the critical current $I_{c}$, of a $S N S$ junction is determined by the occupation of Andreev levels. In equilibrium this occupation is governed by the Fermi distribution function $f_{0}(\epsilon)$. For example, by increasing the temperature $f_{0}(\epsilon)$ broadens. This leads to a occupation of formerly empty higher Andreev levels carrying a supercurrent opposite to the net supercurrent and to an emptying of formerly occupied levels. Altogether, this results in a decrease of the critical current and thus explains the decrease of $I_{c}$ with increasing temperature.

Let us now explore the effect on the supercurrent in an $S N S$ junction when the equilibrium distribution function is replaced by a nonequilibrium distribution function. From the discussion given above it is immediately clear that this directly affects the critical current of the junction. Experimentally, a nonequilibrium distribution function can be realized by injecting hot carriers into the normal conducting area. In case of strong scattering between particles mainly a broader distribution function can be found, which resembles a Fermi distribution function corresponding to a higher 
temperature. ${ }^{20,1}$ Consequently, the critical current monotonously decreases with increasing carrier injection. In case of a weaker quasiparticle scattering a steplike distribution function can be obtained, which deviates largely from the Fermi distribution function. ${ }^{20}$ For this situation even a reversal of the Josephson supercurrent was obtained. ${ }^{12}$ In most experimental situations the distribution function will be in between both extreme limits. ${ }^{2,3}$

In this report we discuss the carrier injection into a ballistic superconductor/two-dimensional electron gas multiterminal junction. The 2DEG is located in a strained InGaAs/ InP layer. The high indium content of $77 \%$ of the channel layer ensures that the mobility is sufficiently large, in order to operate the junction in the ballistic regime. ${ }^{21,22}$ Furthermore, the low Schottky barrier leads to a high transparency at the superconductor/2DEG interface. ${ }^{23}$ Two different modes of injection are employed. First, in the three-terminal configuration the injection current is driven from a normal conducting terminal to one of the superconducting electrodes. Second, in the four-terminal mode the injection current flows between two opposite normal conducting terminals. The effect of current injection on the critical current of the $S / 2 \mathrm{DEG} / S$ junction for both configurations is compared. Additional normal conducting terminals at both injectors were used to determine the potential drop between the superconducting electrodes and the normal conductor. By adjusting the magnetic flux through the junction to the first minimum of the $I_{c}$ vs magnetic field $B$ interference pattern, even an increase of the critical current can be obtained. Most theoretical models describing the carrier injection into an SNS junction are restricted to the one-dimensional case. Here, we present a model, where the effect of an injection into a twodimensional junction is calculated. The theoretical results are finally compared to our experimental findings.

In Sec. II the sample preparation and measurement setup is described. The experimental results are discussed in Sec. III. In Sec. IV the theoretical model for a carrier injection into a two-dimensional junction is presented. The experimental and theoretical results are discussed in Sec. V. The results are concluded in Sec. VI.

\section{EXPERIMENTAL}

The strained $\mathrm{In}_{0.53} \mathrm{Ga}_{0.47} \mathrm{As} / \mathrm{In}_{0.77} \mathrm{Ga}_{0.23} \mathrm{As} / \mathrm{InP}$ layer system used for our samples was grown by metal-organic vapor phase epitaxy. ${ }^{21,22}$ First, a 300-nm-thick InP buffer layer followed by a 9 -nm-thick $n$-doped $\operatorname{InP}(\mathrm{Si})$ layer $\left(N_{D}=4.9\right.$ $\times 10^{17} \mathrm{~cm}^{-3}$ ) was grown. The 10 -nm-thick strained $\mathrm{In}_{0.77} \mathrm{Ga}_{0.23}$ As layer was separated by a 20 -nm-thick InP spacer from the dopant layer. The the structure was finally capped by a 115-nm-thick $\operatorname{In}_{0.53} \mathrm{Ga}_{0.47} \mathrm{As}$ layer. Shubnikov-de Haas experiments performed at a temperature of $0.6 \mathrm{~K}$ revealed a sheet electron concentration of $n=7.68$ $\times 10^{11} \mathrm{~cm}^{-2}$ and a mobility of $\mu=363600 \mathrm{~cm}^{2} / \mathrm{V} \mathrm{s}$. These values result in an elastic mean free path of $l_{e}=5.3 \mu \mathrm{m}$ and a specific sheet resistance of $R_{2 \mathrm{DEG}}^{\square}=22.35 \Omega / \square$. Taking the effective electron mass $m^{*}$ of $0.04 m_{e}$ into account, which was determined by temperature depended Shubnikov-de Haas effect measurements, the phase coherence length for

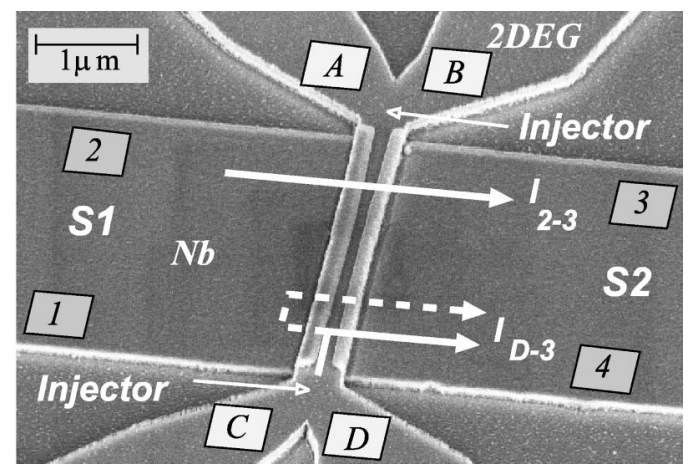

FIG. 1. Scanning electron micrograph of the multiterminal NbInGaAs/InP-Nb junction. The arrows show the current transfer for one of the investigated measurement configurations. The dashed line represents the partial transfer of the injection current through the opposite interface.

the clean limit case can be calculated by using the following expression for the two-dimensional case: $\xi_{c}$ $=\hbar^{2} \sqrt{2 \pi n} / 2 \pi m^{*} k_{B} T$. At a temperature of $T=0.6 \mathrm{~K}$, where most of the measurements were performed, $\xi_{c}$ $=1.3 \mu \mathrm{m}$ is considerably lower than $l_{e}$. This justifies the application of clean limit case for our structures.

For the fabrication of the multiterminal $\mathrm{Nb} / 2 \mathrm{DEG} / \mathrm{Nb}$ structures first Ni/Au:Ge/Ni $(5 \mathrm{~nm} / 90 \mathrm{~nm} / 25 \mathrm{~nm})$ Ohmic contacts alloyed at a temperature of $400^{\circ} \mathrm{C}$ were defined by optical lithography. Subsequently, the geometry of the semiconductor mesa was defined by electron beam lithography and a $\mathrm{Ti}$ etching mask. By using a $\mathrm{CH}_{4} / \mathrm{H}_{2}$ reactive ion etching (RIE) process the semiconductor areas which were not covered by the Ti mask have been etched down to a depth of $200 \mathrm{~nm}$, which is well below the $\operatorname{In}_{0.77} \mathrm{Ga}_{0.23} \mathrm{As}$ channel layer. Before the sample was covered completely by a 100-nm-thick $\mathrm{Nb}$ layer the sample was cleaned by using a $\mathrm{He}$ electron cyclotron resonance plasma source. ${ }^{24}$ In contrast to the more established $\mathrm{Ar}^{+}$sputter cleaning method ${ }^{25,26}$ this procedure damages the semiconductor surface less due to the considerably lower mass of the He atoms. Atomic force microscope studies confirmed that after the He plasma cleaning the surface morphology has not been changed, whereas a moderate $\mathrm{Ar}^{+}$sputter cleaning procedure leads to a formation of In droplets at the surface due to the higher impact of the $\mathrm{Ar}^{+}$ions. The geometry of the $\mathrm{Nb}$ electrodes were defined by a second electron beam lithography step aligned to the mesa structure. An Al mask was prepared by lift-off for the subsequent $\mathrm{SF}_{6}$ RIE process employed for the $\mathrm{Nb}$ etching step. The $\mathrm{Nb}$ layer had a critical temperature of $8.0 \mathrm{~K}$, resulting in a superconducting gap of $\Delta_{0}=1.2 \mathrm{meV}$.

The geometry of the sample is shown in Fig. 1. The $\mathrm{Nb}$ electrodes of width $L=6 \mu \mathrm{m}$ are contacted at the mesa side walls to the InGaAs/InP wire structures of width $d$ $=500 \mathrm{~nm}$. Each of the $\mathrm{Nb}$ electrodes possesses a pair of contacts $(1+2,3+4)$ to allow four-terminal measurements. In addition, two Ohmic contacts on each side of the wire can be used to inject a current into the normal region of the junction or to measure the voltage drop between the superconductor and the 2DEG. Owing to the large separation between the injectors the transport along this direction can be 


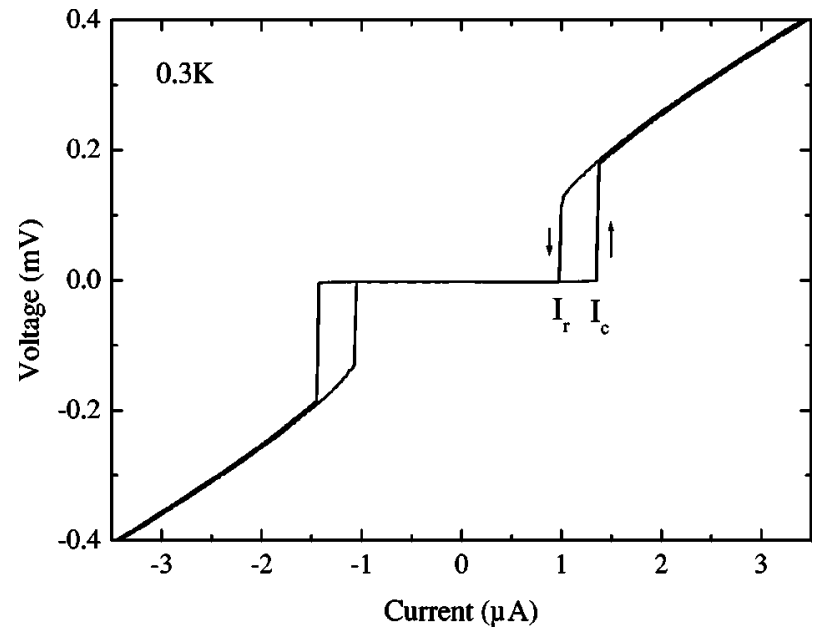

FIG. 2. Current-voltage characteristic of the $S / 2 D E G / S$ junction at a temperature of $0.3 \mathrm{~K}$. The bias current is driven between contacts 2 and 3 while the voltage is measured between terminal 1 and 4.

regarded as diffusive, since $L<t_{e}$, while the transport between the superconducting electrodes is considered to be ballistic.

The measurements are performed in a He-3 cryostat, where the electrical leads are filtered by $\pi$ and RC filters at 300 and $4.2 \mathrm{~K}$, respectively. Additional filtering is gained by $30 \mathrm{~cm}$ long Thermocoax cables, which are directly connected to the sample at low temperature.

\section{EXPERIMENTAL RESULTS}

The basic features of the current-voltage characteristic of the $S / 2 \mathrm{DEG} / S$ junction is shown in Fig. 2. At a temperature of $0.3 \mathrm{~K}$ a critical current of $I_{c}=1.4 \mu \mathrm{A}$ is obtained. At low bias voltages $(<1 \mathrm{mV})$ a normal-state resistance of $R_{N}$ $\approx 110 \Omega$ was determined. This results in a characteristic voltage of $V_{c}=I_{c} R_{N}=150 \mu \mathrm{V}$. As can be seen in Fig. 2, the current-voltage $(I-V)$ characteristics shows a hysteresis. The current $I_{r}=1.0 \mu \mathrm{A}$, where the junction switches back into the superconductive state is considerably lower than the critical current $I_{c}$. As pointed out by Heida, ${ }^{27}$ the hysteresis can be attributed to heating of the two-dimensional electron gas in the heterojunction owing to the voltage drop between the $\mathrm{Nb}$ electrodes.

\section{A. Three-terminal injection}

The decrease of the critical current as a result of a current injection is shown in Fig. 3(a). Here, a three-terminal configuration was chosen, where the injection current is injected from a normal conducting terminal $D$ to a superconducting terminal 3, as illustrated in Fig. 3(b). Owing to the slightly higher temperature of $0.6 \mathrm{~K}$ the current-voltage characteristics shows a smaller critical current of $1.2 \mu \mathrm{A}$ and no hysteresis. The superconductive interval is shifted along the bias-current axis if the injection current is increased. This can be attributed to the fact that the injected carriers are not transferred directly into $\mathrm{Nb}$ electrode $S 2$ but also flow into

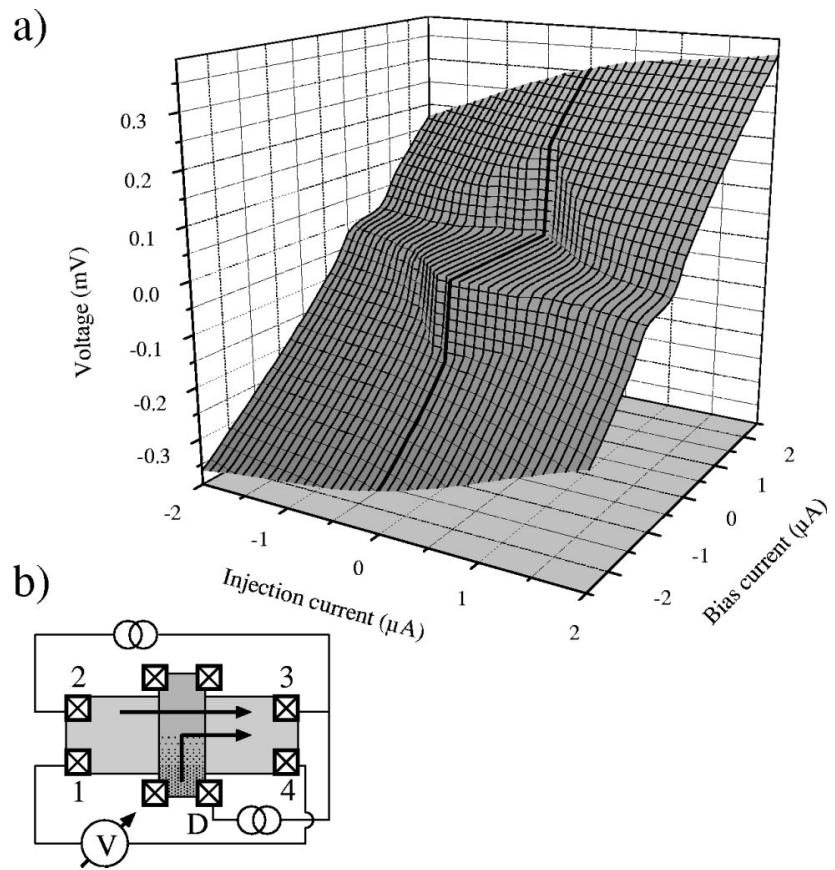

FIG. 3. (a) Suppression of the critical current in the threeterminal configuration. The voltage drop is measured between the superconducting electrodes (1-4) as a function of the junction bias current $I_{2-3}$ for various injection currents between $D-3$. The measurement temperature was $0.6 \mathrm{~K}$. The initial current-voltage characteristic at zero injection current is shown as bold line. (b) Corresponding measurement configuration.

the opposite electrode $S 1$, as illustrated in Fig. $1 .^{2}$ The subsequent carrier transfer from $S 1$ to $S 2$ finally leads to the observed offset contribution.

By an injection in three-terminal configuration the Josephson current cannot be suppressed completely. Up to an injection current of about $I_{D-3}=1.2 \mu \mathrm{A}$ a monotonous decrease of the critical current is observed, as can be seen in Fig. 4. Here, we defined the critical current as $I_{c}=\left(I_{f+}\right.$ $\left.-I_{f-}\right) / 2$, where $I_{f+}$ and $I_{f-}$ correspond to the forward switching current corresponding to a switching of the junc-

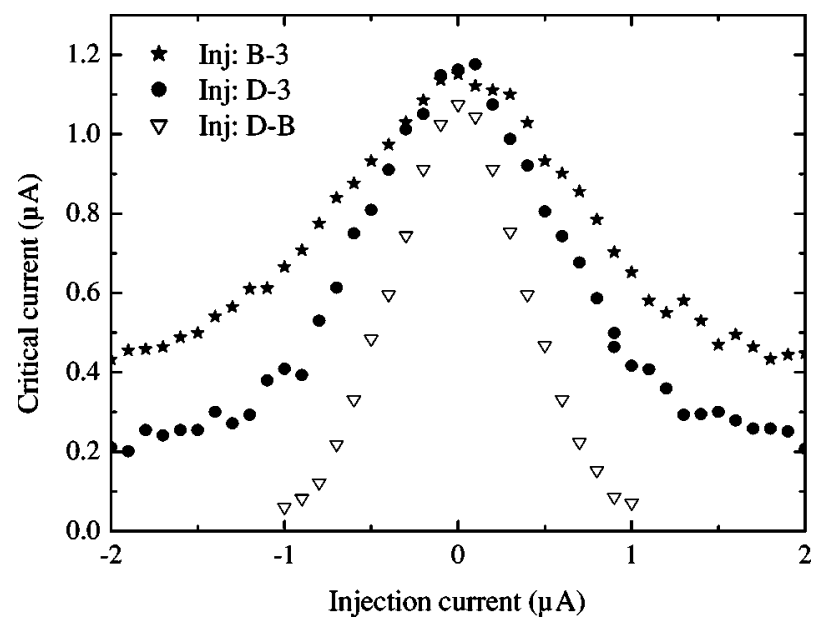

FIG. 4. Critical current as a function of an injection current flowing between terminals $B-3(\star), D-3(\bullet)$, and $D-B(\nabla)$. 


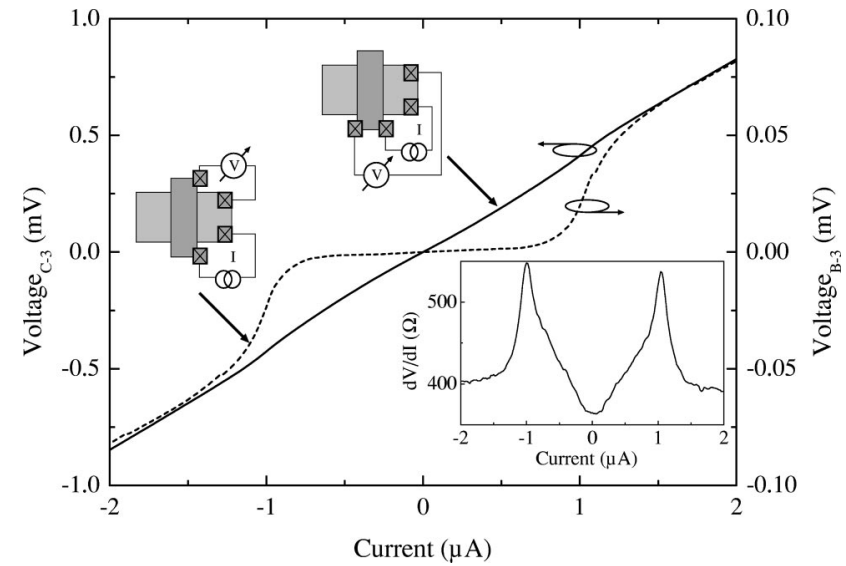

FIG. 5. Voltage drop between contacts $C-3$ (solid line) and 3-B (dashed line) as a function of the injection current driven from terminal $D$ to 4 . The measurement schemes are given in the graph. The inset shows the differential resistance $(C-3)$ as a function of the injection current.

tion from the superconductive state into the normal state for forward and reverse bias current, respectively. In Fig. 4 it can be seen that $I_{c}$ drops to $30 \%$ of its initial value at an injection current $I_{D-3}$ of about $1.2 \mu \mathrm{A}$. At larger injection currents the critical current approaches a constant value. A similar behavior is observed if a current is injected via contact $B$ at the opposite side of the mesa. However, the response of $I_{c}$ on the injection current is weaker in this configuration.

The presence of two contacts at each injector allows us to gain information about the potential difference between the superconducting electrodes and the normal conducting interlayer. The corresponding current-voltage characteristics are shown in Fig. 5. As can be seen here, the voltage measured at the side, where the current is injected $(C-3)$ depends almost linear on the current which is injected from $D$ to 3 . However, the measurement of the differential resistance, shown in Fig. 5 (inset), shows some nonlinearities. First, at large current biases a constant differential resistance of $400 \Omega$ is observed. A comparison with the measurements plotted in Fig. 3 , reveals that for these injection current values the junction is in the normal state. The peaks in the differential resistance found at about $\pm 1 \mu \mathrm{A}$ can be attributed to the transition to the superconductive state of the junction. An interesting feature is the minimum of $d V / d I$ at zero, which is even lower than the differential resistance at large bias currents. We attribute this behavior to the superconductive coupling between the $\mathrm{Nb}$ electrodes and the two-dimensional electron gas.

As long as the $S / 2 \mathrm{DEG} / S$ junction is in the superconducting state no voltage drop is measured between the superconducting electrode and the semiconductor at the opposite side $(B-3)$, as can be seen in Fig. 5. If the junction switches into the normal state at an injection current of about $\pm 0.9 \mu \mathrm{A}$ a finite voltage is observed, which is about one tenth of the voltage measured at the injector side.

In order to elucidate the local effect of the current injection, we compare our experimental results with a calculated potential profile in the normal conductor. If we assume a constant potential in the metallic superconductor and a spe- cific boundary resistance $R_{B}$ of the $S / 2 \mathrm{DEG}$ interface, the following potential profile is obtained:

$$
V(x)=I \frac{L_{\perp}}{d} \frac{R_{2 \mathrm{DEG}}^{\square}}{\sinh \left(L / L_{\perp}\right)} \cosh \left(\frac{x-L}{L_{\perp}}\right) .
$$

Here, $x$ is the distance from the injector, $I$ is the injection current and $L_{\perp}=\sqrt{R_{B} d / 2 R_{2 \mathrm{DEG}}^{\square}}$ is the characteristic length of the current distribution. The corresponding resistance between the injector and the superconducting electrode is given by

$$
R_{3 t}=\frac{V(x=0)}{I}=\frac{L_{\perp} R_{2 \mathrm{DEG}}^{\square}}{d} \operatorname{cotanh} \frac{L}{L_{\perp}} .
$$

From the normal-state resistance $R_{N}$ of the junction we can esitimate the specific boundary resistance: $R_{B} \approx 300 \Omega \mu \mathrm{m}$. According to Eq. (2) this results in a resistance of $R_{3 t}$ $\approx 90 \Omega$ and a characteristic length of $L_{\perp}=1.8 \mu \mathrm{m}$. The theoretically predicted resistance of $R_{3 t}$ is considerably lower than the experimentally observed value of approximately $400 \Omega$. A resistance of about $250 \Omega$ which also higher than the theoretically expected value is found if a current is injected on the opposite side from terminal $B$ to 3 . One possible reason for this discrepancy is that the specific resistance $R_{2 \mathrm{DEG}}^{\square}$ of the 2DEG is about an order of magnitude higher than expected. This might be due to the fact that the electron gas is partly depleted due the metal/semiconductor interface. Another reason might be a decrease of the electron mobility in the 2DEG due to damages caused by the $\mathrm{CH}_{4} / \mathrm{H}_{2}$ reactive ion etching process. A similar effect was observe in resistance measurements on InGaAs/InP quantum wires of comparable width prepared by the same process. ${ }^{28}$ In addition, the difference of the two measured resistance values suggest that the boundary resistance is not homogeneous. From the experimentally obtained values of $R_{3 t}$ we can estimate a value of $L_{\perp}$ which is smaller than one micrometer. This means that the major part of the injection current is transferred within the first half of the junction into the superconducting electrodes. As a consequence, the remaining part of the junction is basically not affected and remains in the superconductive state. ${ }^{3}$

\section{B. Four-terminal injection}

A complete suppression of $I_{c}$ is obtained in a fourterminal configuration where the injection current is driven from one injector to the injector at the opposite side [Fig. 6(a)]. A sketch of the corresponding measurement configuration can be found in Fig. 6(b). A Josephson supercurrent is obtained for injection currents lower than $1.0 \mu \mathrm{A}$. For larger injection currents the junctions is in the normal state. Owing to the four-terminal configuration almost no offset contribution is observed. The remaining small offset can be attributed to a difference in the transparency of the two $\mathrm{Nb} / 2 \mathrm{DEG}$ interfaces. The critical current as a function of the injection current is given in Fig. 4. A striking difference compared to 
a)

b)

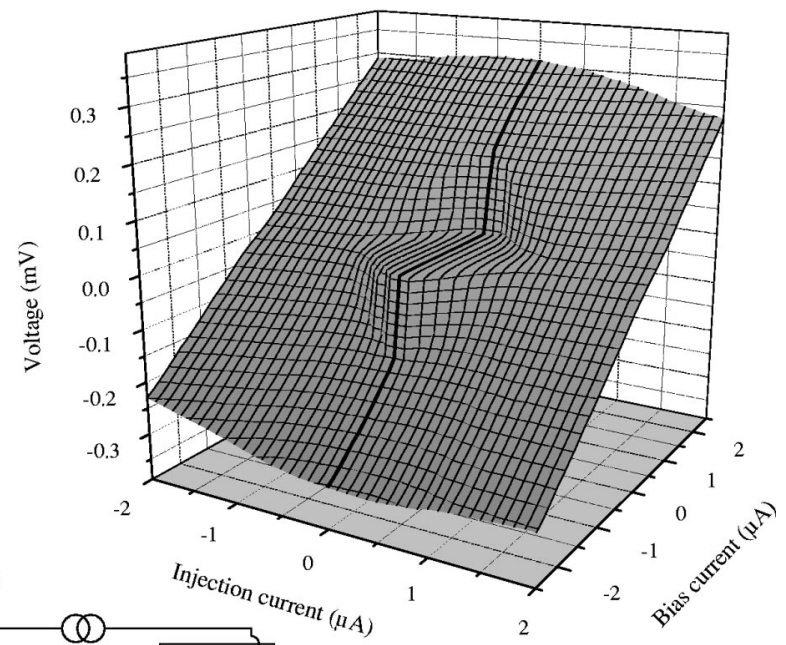

differential resistance shown in Fig. 7 (inset). Two peaks are observed, if the $S / 2 \mathrm{DEG} / S$ junction switches into the normal state. Between these peaks the junction is in the superconductive state and the two superconducting electrodes are at the same potential. In this case the injected current is first almost equally transferred into the two superconducting electrodes and subsequently back into the second injector.

At higher bias currents a resistance of $\approx 520 \Omega$ is found. The voltage drop is not symmetrical between the two injectors. As can be seen in Fig. 7, the voltage measured between the lower injector and the superconducting electrode is higher than for the opposite side, in agreement to the findings related to the three-terminal measurements.

The resistance in the four-terminal configuration which is measured between the two injectors can be estimated by using the following expression: ${ }^{29,30}$

$$
R_{4 t}=\frac{2 L_{\perp} R_{2 \mathrm{DEG}}^{\square}}{d} \tanh \frac{L}{2 L_{\perp}} .
$$

This formula was derived under the same assumptions concerning the interface properties as for the three-terminal case. Inserting the value of $R_{2 \mathrm{DEG}}^{\square}$ of the as-grown heterostructure results in a resistance of $R_{4 t}=172 \Omega$. Once again, this value is too low if it is compared to the measured one. As outlined above, we attribute this disagreement to the increased electron sheet resistance of the 2DEG. However, even if the characteristic length $L_{\perp}$ is in this case smaller than one micrometer, the Josephson supercurrent can be suppressed completely. We can explain this by the fact that the effective area of the junction which is affected by the injected carriers is about twice as large as for the three-terminal configuration.

\section{Recovery of the supercurrent}

The fact that in the three-terminal configuration the junction is only partially affected by the injection current can be used to switch the junction from a linear characteristic to the superconductive state. This is illustrated by the set of measurements shown in Fig. 8. Here, an external magnetic field of $0.12 \mathrm{mT}$ was applied, corresponding to the first minimum in the $I_{c}-B$ Fraunhofer interference pattern, in order to obtain a linear $I-V$ characteristic at zero injection current. Strikingly, a finite supercurrent is recovered if a current is injected from terminal $D$ to 3 ; e.g., for $I_{D-3}=1.0 \mu \mathrm{A}$ a critical current of $0.09 \mu \mathrm{A}$ is obtained. The critical current as function of the injection current is plotted in Fig. 9. It can thus be concluded that an injection current can be used to switch the junction from a linear Ohmic $I-V$ characteristics to a characteristics where a supercurrent appears.

The reappearance of a supercurrent can be explained in the framework of the local suppression of the Josephson supercurrent by the injected carriers. The $I_{c}-B$ measurement of our junction showed an almost ideal Fraunhofer interference pattern so that a homogeneous current density distribution can be assumed along the junction for $B=0$. Thus, for a magnetic field corresponding to the first minimum of the interference the current distribution has a sinusoidal shape. The supercurrent flowing in the center of the junction is can-

FIG. 7. Voltage measured between contacts $D$ and $B$ (solid line) and between $B$ and 3 (dashed line) for a current flowing from one injector to the opposite $(A-C)$. The inset shows the differential resistance measured between the two injectors. 


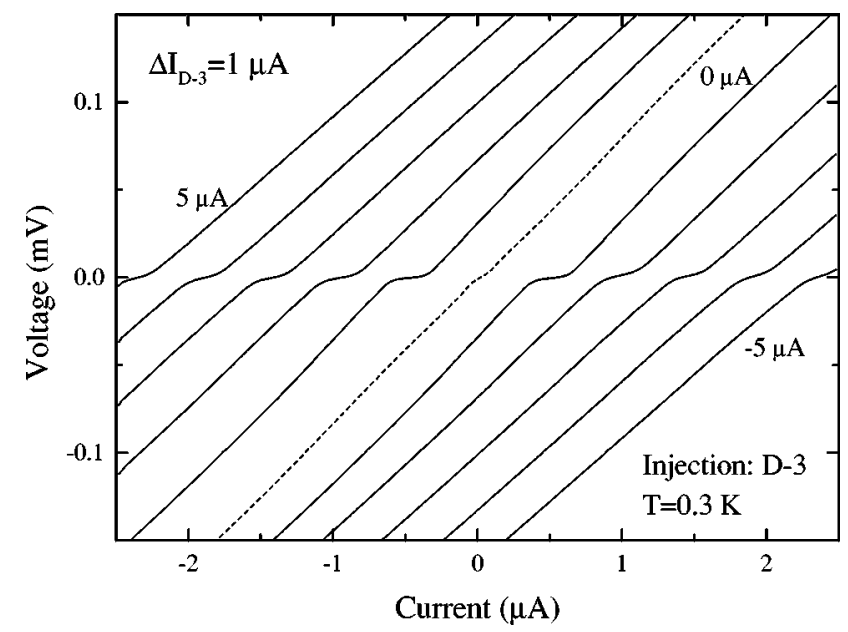

FIG. 8. Current-voltage characteristic of the Josephson junction at $T=0.3 \mathrm{~K}$ with an injection current from terminal $D$ to 3 varied as a parameter. The external magnetic field was adjusted to $0.12 \mathrm{mT}$, which corresponds the first minimum in the $I_{c}$-B-Fraunhofer interference pattern. The field was applied perpendicular to the plane of the 2DEG. The curves are shifted for clarity.

celled exactly by a supercurrent flowing in the opposite direction at the outer areas. By injecting carriers the effective superconductive area of the junction and thus the penetrating magnetic flux is reduced so that the supercurrent is not cancelled anymore. As can be seen in Fig. 9, for injection currents exceeding $1.0 \mu \mathrm{A}$ the critical current decreases again. This effect can be attributed to heating effects.

By injecting carriers from the opposite injector from terminal $B$ to 3 the net supercurrent could also be recovered (Fig. 9). However, a larger injection current is required compared to the injection from the other side $(D-3)$. This observation is in accordance to the measurements of the suppression of the supercurrent at $B=0$. When the current was injected from terminal $B$ to 3 the suppression of the supercurrent was weaker than for an injection from the opposite side (Fig. 4). Thus in the former case the area affected by the injected carriers increases slower with increasing injection current.

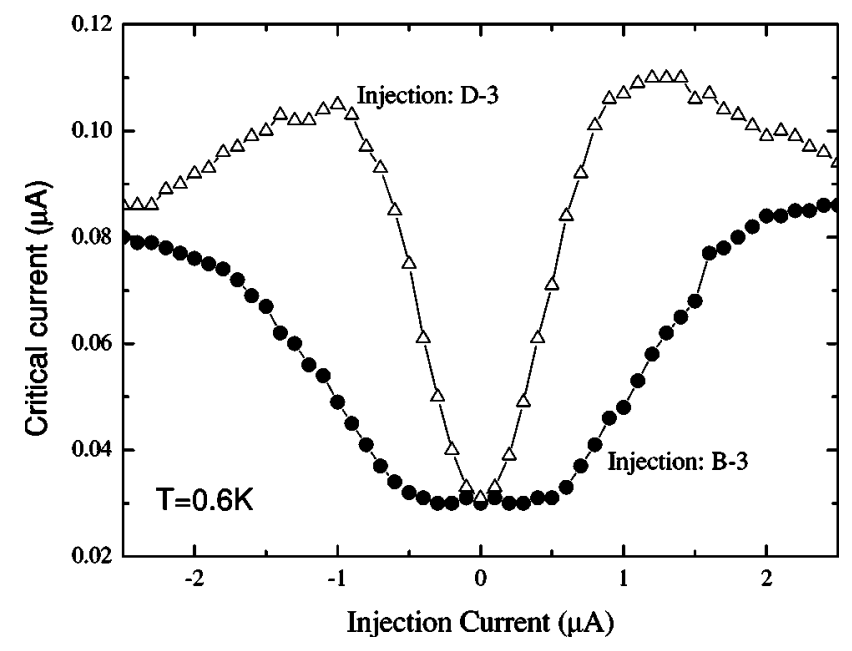

FIG. 9. Critical current as a function of the injection current from terminal $D$ to $3(\triangle)$ and from terminal $B$ to $3(\bullet)$.

\section{THEORETICAL MODEL}

The basic mechanism of critical current control in SNS junctions by current injection is the modification of the occupation of Andreev energy levels with energy $\epsilon<\Delta$ which carry at least a fraction of a supercurrent. $5,6,8,11,13,31$ The strongest effect is expected in short ballistic junctions, where the separation of the superconducting electrodes $d$ is smaller than the clean limit coherence length: $d<\xi_{c}$. Here, a supercurrent is fully determined by the occupation of two Andreev bound states. In longer junctions with $d>\xi_{c}$ a part of the supercurrent is carried by extended states at $\epsilon>\Delta$ which are in equilibrium with the superconducting electrodes of the junction.

The theoretical analysis of the current injection is based on the calculation of the energy dependence of the following two quantities: (a) the distribution function and (b) the socalled spectral current density. As mentioned above, we consider the transport along the wire to be diffusive with an elastic mean free path $l_{e}$ much smaller than the distance $L$ between the two reservoirs attached to the normal conductor. Furthermore, the distribution function in the control line of the mesoscopic junction with $L$ much smaller than the inelastic mean free path $l_{\text {in }}$ is essentially nonequilibrium, as discussed in (Refs. 6,13,20) and may be simply expressed in terms of the Fermi functions of the reservoirs $f_{0}(\epsilon \pm e V / 2)$ with chemical potentials shifted by $\pm e V / 2$. There is no simple expression for the spectral current density in $S N S$ junction in a general case, since it depends on the distance between the electrodes, the impurity concentration and the transparency of the NS interfaces. However, its generic properties are well understood from the previous theoretical work. The limit of a long diffusive $S N S$ junction was considered in (Refs. 11,13), while the clean $S N S$ junction with arbitrary length and a scattering center within $N$ region was studied in (Refs. 6-8). Motivated by the fact that the present $S / 2 \mathrm{DEG} / S$ junctions are very close to the short ballistic regime, while having a finite transparency of the $S / 2 D E G$ interfaces, we shall first demonstrate the theoretical predictions for the case of SINIS junction. Here, I stands for the barrier between the superconductor and normal conductor.

Equilibrium supercurrents in ballistic SINIS junctions were studied theoretically in (Refs. 32-35). In general, an interplay between Andreev and transmission (Breit-Wigner) resonances takes place in these junctions. In Ref. 35 the universal expression for the equilibrium supercurrent was derived in the quasiclassical regime $k_{F} d \gg 1$ by integration over the transmission resonances. In the nonequilibrium situation the general expression for the supercurrent density has the form $^{36}$

$$
J_{s}(\varphi)=\int_{-\infty}^{+\infty} \operatorname{Im} J_{s}(\varphi, \epsilon)[1-2 f(\epsilon)] d \epsilon,
$$

where $\varphi$ is the phase difference across the junction, $f(\epsilon)$ is the nonequilibrium distribution function as a function of energy $\epsilon$ [in equilibrium $1-2 f(\epsilon)=\tanh \epsilon / 2 T]$, and $J_{s}(\varphi, \epsilon)$ is the so-called spectral supercurrent density given by the analytical continuation of the equilibrium supercurrent. ${ }^{35}$ 
In a two-dimensional case, the density of spectral supercurrent density through a ballistic SINIS contact (the mean free path $l_{e} \gg d, d$ is the interlayer thickness) is expressed through the Green's function $G\left(\mathbf{r}, \mathbf{r}^{\prime}\right)$

$$
\operatorname{Im} J_{S}(\varphi, \epsilon)=\operatorname{Im} \frac{i \hbar e}{m} \int \frac{d k_{y}}{2 \pi} \lim _{x^{\prime} \rightarrow x}\left(\frac{\partial}{\partial x^{\prime}}-\frac{\partial}{\partial x}\right) G\left(x, x^{\prime} ; k_{y}, \boldsymbol{\epsilon}\right) .
$$

Here $x, x^{\prime}$ are the coordinates across the junction, $k_{y}$ is the wave vector component normal to the current direction. The normal and the anomalous Green's functions $G\left(x, x^{\prime}\right), F^{+}\left(x, x^{\prime}\right)$ obey the Gor'kov equations

$$
\left(\begin{array}{cc}
\epsilon+H & \widetilde{\Delta}(x) \\
\widetilde{\Delta}^{*}(x) & \epsilon-H
\end{array}\right)\left(\begin{array}{c}
G \\
F^{+}
\end{array}\right)=\left(\begin{array}{c}
\delta\left(x-x^{\prime}\right) \\
0
\end{array}\right),
$$

where $\widetilde{\Delta}=\Delta \exp (i \chi)$ is the spatially dependent complex pair potential, $\quad H=\left(\hbar^{2} / 2 m\right)\left(\partial^{2} / \partial x^{2}\right)+E_{x}-V(x), \quad E_{x}=E_{F}$ $-\hbar^{2} k_{y}^{2} / 2 m$ is the electron kinetic energy across the junction, $E_{F}$ is the Fermi energy, $V(x)=W_{1} \delta(x)+W_{2} \delta(x-d)$ is the interface potential, and $W_{1,2}$ being the barrier strengths.

Matching the solutions of Gor'kov equations in the $N$ and $S$ layers, in the case of a thin interlayer $d<\xi_{c}$ and symmetric low-transparent barriers $W_{1,2}=W,\left(W / \hbar v_{F}\right) \gg 1$, one arrives the following expression for the spectral supercurrent $J_{s}:{ }^{35}$

$$
\operatorname{Im} J_{S}(\varphi, \epsilon)=\frac{e}{\hbar} \int \frac{d k_{y}}{2 \pi} \operatorname{Re} \frac{\Delta_{0}^{2} \sin \varphi}{2 \widetilde{W}^{4} E_{1}^{2}\left(\cos d / \xi_{x}-\cos 2 k_{x} d\right)+E_{3}^{2}} .
$$

Here, $\varphi$ is the phase difference across the junction, $\Delta_{s}$ is the pair potential in $S, E_{1}=\sqrt{\Delta_{0}^{2}-\epsilon^{2}}, E_{3}=\sqrt{\Delta_{0}^{2} \cos ^{2} \varphi / 2-\epsilon^{2}}$, $\widetilde{W}=W / \hbar v_{x}, \quad k_{x}=k_{F} \cos \theta, \quad v_{x}=v_{F} \cos \theta, \quad$ and $\quad \xi_{x}(\epsilon)$ $=\xi_{N}(\epsilon) \cos \theta=\hbar v_{F} \cos \theta / 2 \epsilon$ is the coherence length in the interlayer along the interface normal. The above expression has a resonant structure. The integration over the directions of $k_{y}$, i.e., over the transmission (Breit-Wigner) resonances, yields the total supercurrent and depends on the relation between the resonance width and the superconducting energy gap.

In order to introduce the transmission resonances, we start with the expression for the transmission coefficient of a junction in a normal state $D_{N}$ :

$$
D_{N}^{-1}=1+\left(2 \widetilde{W} \cos k_{x} d+2 \widetilde{W}^{2} \sin k_{x} d\right)^{2} .
$$

The width of the resonances is given by $\Gamma$ $=\hbar v_{F}\langle u D(u)\rangle / 2 d=2 \hbar^{3} v_{F}^{3} /\left(3 d W^{2}\right)$, where $\langle u D(u)\rangle$ is the angle-averaged single-electron transmission coefficient of an individual barrier and $u=\cos \theta$. In the low-transparency regime $D(u)=\left(\hbar v_{F} u / W\right)^{2}$.

Below we consider the quasiclassical regime $k_{F} d \gg 1$, when an integration over sharp resonances can be performed first. In a two-dimensional (2D) case, which we are interested in here, the spectral supercurrent density is given by

$$
\operatorname{Im} J_{s}(\varphi, \epsilon)=\frac{e k_{F}}{4 \pi^{2} \hbar} \frac{\Delta_{0}^{2} \sin \varphi}{\Delta_{0}^{2}-\epsilon^{2}} \operatorname{Re} \int_{-\pi / 2}^{\pi / 2} \frac{\cos ^{3} \theta d \theta}{\widetilde{W}^{2} \sqrt{a^{2}-1}},
$$

where $\quad a(\varphi, \epsilon)=\cos \left(d / \xi_{n x}\right)+\left(1 / 2 \widetilde{W}^{4}\right)\left(\Delta_{0}^{2} \cos ^{2} \varphi / 2-\epsilon^{2}\right) /\left(\Delta_{0}^{2}\right.$ $-\epsilon^{2}$ ). In the ballistic regime the specific resistance of the junction in the normal state is twice the resistance of $\mathrm{Nb} /$ 2DEG interface $R_{2 \mathrm{D}}=2 R_{B}$ :

$$
\begin{gathered}
R_{2 \mathrm{D}}^{-1}=\frac{1}{2} \frac{e^{2} k_{F}}{2 \pi^{2} \hbar}\langle u D(u)\rangle \\
=\frac{1}{2} \frac{e^{2} k_{F}}{2 \pi^{2} \hbar} \int_{-\pi / 2}^{\pi / 2} \frac{\hbar^{2} v_{F}^{2} \cos ^{3} \theta d \theta}{W^{2}}=\frac{e^{2} k_{F}}{3 \pi \hbar}\left(\frac{\hbar v_{F}}{W}\right)^{2} .
\end{gathered}
$$

Finally the normalized spectral supercurrent density can be written in the form

$$
\operatorname{Im} J_{s}(\varphi, \epsilon) e R_{2 \mathrm{D}}=\frac{3}{2} \frac{\Delta_{0}^{2} \sin \varphi}{\Delta_{0}^{2}-\epsilon^{2}} \operatorname{Re} \int_{0}^{\pi / 2} \frac{\cos ^{3} \theta d \theta}{\widetilde{W}^{2} \sqrt{a^{2}-1}} .
$$

In the coherent regime (broad resonances) $\Gamma>\Delta_{0}$ we get $\sqrt{a^{2}-1} \simeq \widetilde{W}^{-2}\left(\Delta_{0}^{2} \cos ^{2} \varphi / 2-\epsilon^{2}\right)^{1 / 2}\left(\Delta_{0}^{2}-\epsilon^{2}\right)^{-1 / 2}$ and the general result Eq. (9) is reduced to the simple expression

$$
\operatorname{Im} J_{s}(\varphi, \epsilon) e R_{2 \mathrm{D}}=\frac{\theta\left(\Delta_{0}-\epsilon\right) \theta\left(\epsilon-\Delta_{0} \cos \varphi / 2\right) \Delta_{0}^{2} \sin \varphi}{\sqrt{\Delta_{0}^{2}-\epsilon^{2}} \sqrt{\epsilon^{2}-\Delta_{0}^{2} \cos ^{2} \varphi / 2}} .
$$

A similar result was obtained earlier in Ref. 35 for the 3D case. Thus, according to Eq. (13), the spectral supercurrent density in the coherent regime has a universal energy dependence, independently of the properties of the interlayer and of the contact dimensionality. The spectral supercurrent is nonzero only in the range $\Delta_{0} \cos \varphi / 2<\epsilon<\Delta_{0}$, i.e., there is a minigap $\Delta_{0} \cos \varphi / 2$ in the spectrum of the Andreev bound states. On the other hand, all states in the energy range $\Delta_{0} \cos \varphi / 2<\epsilon<\Delta_{0}$ contribute to the supercurrent. That means that the contact is in the intermediate regime between a short ballistic weak link (bound state energy $\Delta_{0} \cos \varphi / 2$ ) and a tunnel junction (bound state energy $\Delta_{0}$ ). ${ }^{37,35}$ Physically this is due to the properties of the distribution of transmission eigenvalues in a double-barrier junction, which is a combination of open and closed channels. ${ }^{35}$

In the incoherent regime $\Gamma \ll \Delta_{0}$ this universality breaks down due to a dephasing of the transmission resonances. Then the current-phase relation is sinusoidal and the critical current scales as $J_{c}(\varphi, \epsilon) \sim \Delta_{0}^{2}\left(\Delta_{0}^{2}-\epsilon^{2}\right)^{-1} / \gamma_{\text {eff }}$, with $\gamma_{\text {eff }}$ $=\pi k_{B} T_{c} / \Gamma=2 \pi k_{B} T_{c s} d /\left[\hbar v_{F}\langle u D(u)\rangle\right] \gg 1$. In this case there is no minigap in the spectrum of the Andreev bound states of the junction.

The above results are illustrated by the numerical calculations of $J_{s}(\varphi, \epsilon) e R_{2 \mathrm{D}}$ presented in Fig. 10 for two different values of transmission probability across the single barrier for $D_{0}=1 /\left(1+\widetilde{W}^{2}\right)$. As a reference scales, we have introduced the coherence length $\xi_{0}=\hbar v_{F n} / 2 \Delta_{0}$, where $v_{F n}$ is the Fermi velocity in the normal conductor. The parameter $\gamma_{\text {eff }}$ 

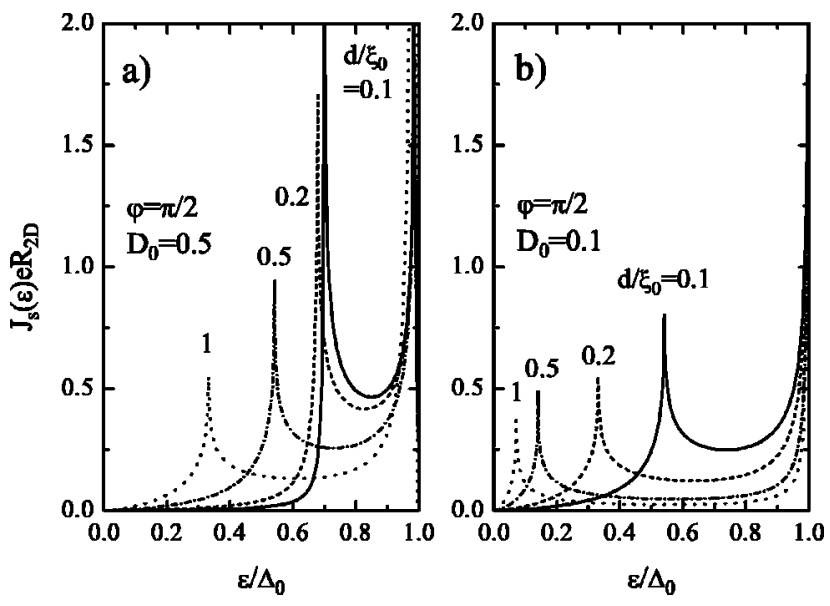

FIG. 10. Normalized spectral supercurrent density as a function of energy for various values of the ratio $d / \xi_{0}$. The phase difference between the superconductive electrodes was fixed at $\varphi=\pi / 2$ (a) Results for an interface transparency of $D=0.5$, (b) results for $D$ $=0.1$.

of the model is then expressed as $\gamma_{\mathrm{eff}}=(4 d) /\left(3 \xi_{c}^{*} D_{0}\right)$, with $\xi_{c}^{*}=\left(\gamma_{E} / \pi\right) \xi_{0} \simeq \xi_{c}^{*} / 1.76$ where $\gamma_{E} \simeq 1.78$ is the Euler's constant. It is seen that with increasing $\gamma_{\text {eff }}$ the sharp gap in the spectrum disappears and low-energy states are gradually filled in. On the other hand, in the regime of small $\gamma_{\text {eff }}$ the spectral supercurrent becomes very close to the limiting twopeak behavior given by Eq. (13).

According to Eq. (4), these features of the spectral supercurrent manifest itself in the dependence of $I_{s}(\varphi)$ vs voltage. In the four-terminal configuration the nonequilibrium distribution within the interlayer can be approximated by $f(\epsilon)$ $=1 / 2 f_{0}(\epsilon-e V / 2)+1 / 2 f_{0}(\epsilon+e V / 2)$. The results of calculations are presented in Fig. 11.

In contrast to calculations on one-dimensional systems, ${ }^{38}$ the supercurrent monotonously decreases with increasing in-

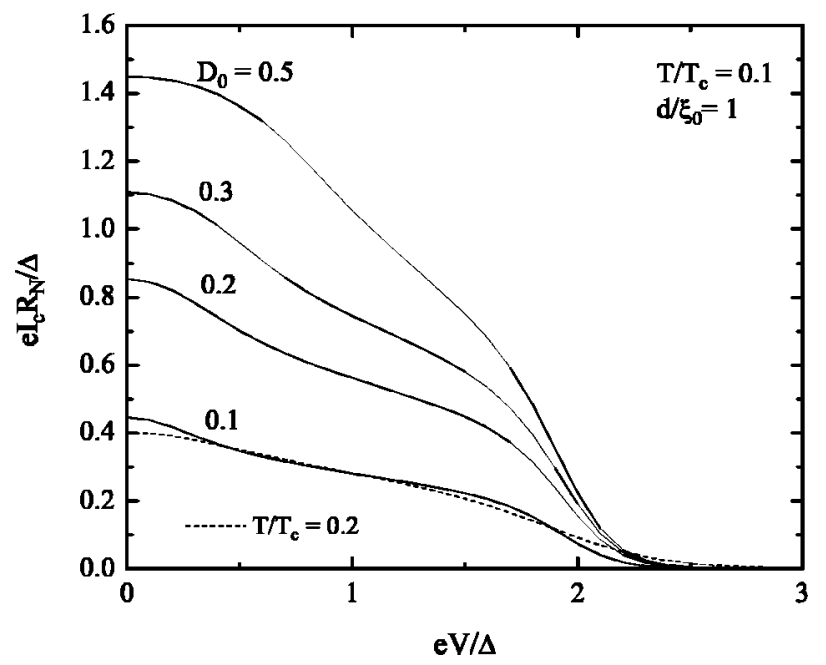

FIG. 11. Calculated suppression of the Josephson supercurrent in a four-terminal configuration as a function of voltage between the two injector contacts. The interface transparency $D_{0}$ was varied from 0.1 to 0.5 . The ratio $d / \xi_{0}$ was fixed at 1 . jection voltage $e V / \Delta_{0}$. A full suppression is obtained at approximately $e V=2 \Delta_{0} \cdot{ }^{39}$ If the applied voltage $e V$ matches the position of the peaks in the spectral supercurrent density (Fig. 10), the decrease of the critical current is larger leading to a two-step profile. Owing to the large density of Andreev bound states at this position, an occupation or a depletion of the Andreev bounds states results in a large change of the net supercurrent. If the interface transparency $D_{0}$ is decreased the total supercurrent is decreased. Since the peak in the spectral supercurrent density are shifted towards lower energies, the steps are found at lower bias voltages accordingly. As can be seen in Fig. 11 (dashed line), the steps are washed out completely if the temperature is increased.

\section{DISCUSSION}

Let us first qualitatively compare the experimental results with the model. Only the measurements performed in the four-terminal configuration can be compared, since no current transfer from the injector into the superconducting electrodes is included in the model. The basic features found in the four-terminal measurements are reproduced by the model. The critical current monotonously decreases with increasing voltage applied between the injectors. In contrast to a one-dimensional model where a discrete Andreev level spectrum is obtained a continuous level spectrum is found if a two-dimensional junction is regarded. Especially in the incoherent regime $\left(\Gamma \ll \Delta_{0}\right)$, the spectral supercurrent density is nonzero in the whole range within the superconductive gap, as can be seen in Fig. 10. As a consequence, even small injection currents result in a depletion/occupation of Andreev bound states and thus to a decrease of the critical current.

As mentioned above, in the model a complete suppression of the supercurrent is obtained for $e V \approx 2 \Delta_{0}$. As can be taken from Fig. 7, a complete suppression of the supercurrent is achieved for an injection current of approximately $1 \mu \mathrm{A}$. This corresponds to a voltage drop between the injectors of about $0.6 \mathrm{mV}$, which is considerably lower than $2 \Delta_{0} / e$.

In order to find an explanation for this discrepancy we need to determine some parameters from the experimental results which are relevant in the model. By taking the value of the sheet electron concentration a Fermi wave number of $220 \times 10^{6} 1 / \mathrm{m}$ is obtained, which results in $k_{F} d=110 \gg 1$, as required for the quasiclassical regime described by the model. From measurements of the differential resistance as a function of the bias voltage at a single $\mathrm{Nb} / 2 \mathrm{DEG}$ interface, which were prepared on the same chip, an interface transparency $D_{0}$ of 0.6 was extracted, which is close to the maximum value of $D_{0}=0.5$ considered in the model. The temperature-independent clean-limit coherence length $\xi_{c}^{*}$ has a value of $100 \mathrm{~nm}$ for our sample. This results in a value of 2.5 for $d / 2 \xi_{c}^{*}$. The latter quantity is a measure of the number of Andreev bound states in the direction perpendicular to the $S / 2 D E G$ interface. However, our model is restricted to the case where $d / 2 \xi_{c}^{*}<1$.

The fact that for our sample the quantity $d / 2 \xi_{c}^{*}$ is larger than one might be the reason, why the supercurrent is suppressed at lower injection voltages. Our junction can be considered to be in the intermediate range between the short and 
long-junction regime. In contrast to our model, probably more than one Andreev level is present perpendicular to the $S / 2 \mathrm{DEG}$ interface. Due to the larger number of Andreev levels within the gap, the Andreev levels which carry a supercurrent in the opposite direction are closer to the Fermi energy. As a consequence, the supercurrent is decreased at lower injection voltages. At higher injection currents, an effective heating of the electron gas between the two superconducting electrodes might enhance the suppression of the supercurrent.

\section{CONCLUSIONS}

In conclusion, control of the supercurrent of a $S / 2 \mathrm{DEG} / S$ junction by injection hot carriers was studied. In a fourterminal configuration a complete suppression of the critical current was achieved. In contrast, in a three-terminal configuration, the Josephson supercurrent could not be suppressed completely. An analysis of the resistances measured between the injector contact and one of the superconducting electrodes revealed that most of the injection current was transferred to the $\mathrm{Nb}$ electrodes in the vicinity of the injector.
As a consequence, in the three-terminal configuration a significant part of the junction is not affected by the injected carriers. In order to explain our experimental results, a theoretical model was introduced which explicitly takes the twodimensional character of our junction into account. In agreement to the experiments a monotonous decrease of the critical current with increasing injection current was found in the calculations. However, the injection voltages required to suppress the supercurrent completely were considerably lower than predicted by the model. We attributed this to fact that our junction is in the intermediate regime between a short and long junction, while our model only covers the short junction regime.

\section{ACKNOWLEDGMENTS}

The authors thank A. van der Hart for the electron beam lithography and G. Müllejans and H. Kertz for their excellent assistance during the measurements. This work was supported by the Deutsche Forschungsgemeinschaft (DFG) contract SCHA 835 1-1.
*Electronic address: th.schaepers@fz-juelich.de

†Present address: Infineon Technologies Dresden P.O. Box 1009 40, D-01076 Dresden, Germany.

${ }^{\ddagger}$ Present address: G.L.I. Global Light Industries GmbH, D-47475 Kamp-Lintfort, Germany.

${ }^{1}$ A. F. Morpurgo, T. M. Klapwijk, and B. J. van Wees, Appl. Phys. Lett. 72, 966 (1998).

${ }^{2}$ Th. Schäpers, J. Malindretos, K. Neurohr, S. G. Lachenmann, A. van der Hart, G. Crecelius, H. Hardtdegen, H. Lüth, and A. A. Golubov, Appl. Phys. Lett. 73, 2348 (1988).

${ }^{3}$ K. Neurohr, Th. Schäpers J. Malindretos, S. Lachenmann, A. I. Braginski, H. Lüth, M. Behet, G. Borghs, and A. A. Golubov, Phys. Rev. B 59, 11197 (1999).

${ }^{4}$ A. Richter, Adv. Solid State Phys. 40, 321 (2000).

${ }^{5}$ B. J. van Wees, K.-M. H. Lenssen, and C. J. P. M. Harmans, Phys. Rev. B 44, 470 (1991).

${ }^{6}$ P. Samuelsson, V. S. Shumeiko, and G. Wendin, Phys. Rev. B 56, 5763 (1997).

${ }^{7}$ P. Samuelsson, J. Lantz, V. S. Shumeiko, and G. Wendin, Phys. Rev. B 62, 1319 (2000).

${ }^{8}$ L.-F. Chang and P. F. Bagwell, Phys. Rev. B 55, 12678 (1997).

${ }^{9}$ H. T. Ilhan and P. F. Bagwell, J. Appl. Phys. 84, 6758 (1998).

${ }^{10}$ H. T. Ilhan, H. V. Demir, and P. F. Bagwell, Phys. Rev. B 58, 15120 (1998).

${ }^{11}$ A. F. Volkov, Phys. Rev. Lett. 74, 4730 (1995).

${ }^{12}$ J. J. A. Baselmans, A. F. Morpurgo, B. J. van Wees, and T. M. Klapwijk, Nature (London) 397, 43 (1999).

${ }^{13}$ F. K. Wilhelm, G. Schön, and A. D. Zaikin, Phys. Rev. Lett. 81, 1682 (1998).

${ }^{14}$ J. Kutchinsky, R. Taboryski, Claus B. Sørensen, J. B. Hansen, and P. E. Lindelof, Phys. Rev. Lett. 83, 4856 (1999).

${ }^{15}$ I. O. Kulik, Zh. Éksp. Teor. Fiz. 57, 1745 (1969) [Sov. Phys. JETP 30, 944 (1970)]

${ }^{16}$ C. Ishii, Prog. Theor. Phys. 5, 1525 (1972).

${ }^{17}$ J. Bardeen and J. L. Johnson, Phys. Rev. B 5, 72 (1971).
${ }^{18}$ H. X. Tang, Z. D. Wang, and Y. Zhang, Z. Phys. B: Condens. Matter 101, 359 (1996).

${ }^{19}$ A. F. Andreev, Zh. Éksp. Teor. Fiz. 461823 (1964) [Sov. Phys. JETP 19, 1228 (1964)].

${ }^{20}$ H. Pothier, S. Guèron, N. O. Birge, D. Esteve, and M. H. Devoret, Phys. Rev. Lett. 79, 3490 (1997).

${ }^{21}$ H. Hardtdegen, R. Meyer, H. Løken-Larsen, J. Appenzeller, Th. Schäpers, and H. Lüth, J. Cryst. Growth 116, 521 (1992).

${ }^{22}$ H. Hardtdegen, R. Meyer, M. Hollfelder, Th. Schäpers, J. Appenzeller, H. Løken-Larsen, Th. Klocke, Ch. Dieker, B. Lengeler, H. Lüth, and W. Jäger, J. Appl. Phys. 73, 4489 (1993).

${ }^{23}$ K. Neurohr, A. A. Golubov, Th. Klocke, J. Kaufmann, Th. Schäpers, J. Appenzeller, D. Uhlisch, A. V. Ustinov, M. Hollfelder, H. Lüth, and A. I. Braginski, Phys. Rev. B 54, 17018 (1996).

${ }^{24}$ Th. Schäpers, R. P. Müller, G. Crecelius, H. Hardtdegen, and H. Lüth, J. Appl. Phys. 88, 4440 (2000).

${ }^{25}$ H. Takayanagi, T. Akazaki, J. Nitta, and T. Enoki, Jpn. J. Appl. Phys. 34, 1319 (1995).

${ }^{26}$ Th. Schäpers, A. Kaluza, K. Neurohr, J. Malindretos, G. Crecelius, A. van der Hart, H. Hardtdegen, and H. Lüth, Appl. Phys. Lett. 71, 3537 (1997).

${ }^{27}$ J. P. Heida, Ph.D. thesis, Rijksuniversiteit Groningen, The Netherlands, 1998.

${ }^{28}$ A. Kaluza, Master's thesis, Technische Hochschule Aachen, RWTH, 1996.

${ }^{29}$ M. Yu. Kupriyanov, Sov. J. Supercond. 2, 5 (1992).

${ }^{30}$ D. Uhlisch, Ber. Forschungszent. Juelich 3490, 1 (1998).

${ }^{31}$ S.-K. Yip, Phys. Rev. B 58, 5803 (1998).

${ }^{32}$ A. Furusaki, H. Takayanagi, and M. Tsukada, Phys. Rev. B 45, 10563 (1992).

${ }^{33}$ A. Chrestin, T. Matsuyama, and U. Merkt, Phys. Rev. B 49, 498 (1994).

${ }^{34}$ A. L. Gudkov, M. Yu. Kupriyanov, and K. K. Likharev, Zh. Éksp. Teor. Fiz. 94, 319 (1988) [Sov. Phys. JETP 67, 1478 (1988)]. 
${ }^{35}$ A. Brinkman and A. A. Golubov, Phys. Rev. B 61, 11297 (2000).

${ }^{36}$ A. I. Larkin and Yu. N. Ovchinnikov, Zh. Éksp. Teor. Fiz. 73, 299 (1977) [Sov. Phys. JETP 46, 155 (1977)].

${ }^{37}$ M. Yu. Kupriyanov and V. F. Lukichev, Zh. Éksp. Teor. Fiz. 94, 139 (1988) [Sov. Phys. JETP 67, 1163 (1988)].
${ }^{38}$ A. A. Golubov, K. Neurohr, Th. Schäpers, M. Behet, and H. Lüth, Superlattices Microstruct. 25, 1033 (1999).

${ }^{39}$ In our numerical calculations the relation $D(\theta)=D_{0} \cos ^{2} \theta /(1$ $+D_{0} \cos ^{2} \theta$ ) was approximated by $D(\theta)=D_{0} \cos ^{2} \theta$ (assuming small $D_{0}$ ). 